\title{
Klinische Bedeutung des gastroösophagealen Refluxes beim Asthma bronchiale
}

\author{
Clinical Significance of Gastrooesophageal Reflux in Asthma
}

Autoren

Institute
T. Rothe ${ }^{1}$, R. Buhl' ${ }^{2}$, A. Gillissen ${ }^{3}$, P. Kardos ${ }^{4}$, K. F. Rabe ${ }^{5}$, R. Sauer ${ }^{6}$, C. Vogelmeier ${ }^{7}$, H. Worth ${ }^{8}$, R. Arnold ${ }^{9}$, G. Menz ${ }^{10}$

Die Institutsangaben sind am Ende des Beitrags gelistet. eingereicht 29. 5. 2006

akzeptiert 17. 7. 2006

Bibliografie

DOI $10.1055 / \mathrm{s}-2006-944272$

Pneumologie 2006; 60; 672-678

(c) Georg Thieme Verlag KG

Stuttgart · New York

ISSN 0934-8387

Korrespondenzadresse PD Dr. Günter Menz Hochgebirgsklinik Davos 7265 Davos-Wolfgang

\section{Zusammenfassung}

Im klinischen Alltag koinzidieren asthmatische Beschwerden und gastroösophagealer Reflux (GÖR) häufig. Bei der Behandlung einer gastroösophagealen Refluxkrankheit muss ein parallel bestehendes Asthma dann nicht mitberücksichtigt werden, wenn das Asthma gut kontrolliert ist. Im Falle eines symptomatischen Asthmas, das auch auf eine Therapieintensivierung nicht, oder nur unzureichend anspricht, besteht jedoch die Möglichkeit, dass der GÖR und die Therapierefraktärität des Asthmas in einem kausalen $\mathrm{Zu}$ sammenhang stehen. Ein Algorithmus soll in diesem Fall das klinische Vorgehen leiten.

\section{Abstract}

In clinical practice, gastrooesophageal reflux (GER) and bronchial asthma often coincide. In the case of sufficient asthma control, the asthma does not need to be taken into account when treatment of GER is being evaluated. In patients with symptomatic asthma despite adequate antiasthmatic treatment, a possible causal relationship between GER and the poor responsiveness to asthma therapy has to be considered. An algorithm to guide the diagnostic and therapeutic steps in such cases is presented.

\section{Einleitung}

Ein potenzieller Zusammenhang zwischen einem gastroösophagealen Säurereflux und Asthmasymptomen wird seit mehr als einem Jahrhundert diskutiert. Vor allem in den letzten Jahren sind zu diesem Thema sehr viele, teils kontroverse Publikationen entstanden. Der nachfolgende Artikel stellt eine Konsensmeinung der Autoren dar, die das Thema im Rahmen der 8. Davoser Gespräche am 12. und 13. Januar 2006 diskutiert haben.

\section{Epidemiologie}

GÖR, der retrograde Übertritt von Magensäure in den Ösophagus, tritt physiologischerweise postprandial, vor allem tagsüber, aber auch spontan auf. Er wird charakteristischerweise vom Patienten nicht bemerkt und ist Folge spontaner Relaxationen des unteren Ösophagussphinkters (TLESR $=$ Transient Lower Esophageal Relaxations). Beim Gesunden sind 40-60\% der TLESRs, beim Refluxpatienten $60-70 \%$ der TSLERs mit
GÖR assoziiert. Dagegen sind nur 5-10\% der Refluxepisoden Folge schluckbedingter Relaxationen des unteren Ösophagussphinkters. Pathologischer, d.h. über das physiologische Maß vermehrter GÖR, wird in vielen Fällen durch Sodbrennen bzw. schwallartiges Aufstoßen von Magensäure und zerkauten Nahrungsbestandteilen (Regurgitationen) wahrgenommen. Er lässt sich durch die Messung der Säureexposition des Ösophagus (24-Stunden pH-Metrie) quantifizieren. Pathologisch vermehrter GÖR ist oft mit einer Hiatushernie assoziiert.

Mehrere klinische Studien [1-3], die auf 24-Stunden-pH-Messungen beruhen, lassen erkennen, dass die Sensitivität und Spezifität des Symptoms „Sodbrennen“ zur Erkennung von GÖR ungenügend ist; ein Umstand, dem im klinischen Alltag Rechnung getragen werden muss. So korreliert das subjektive Empfinden von Sodbrennen nur mäßig mit dem gemessenen Abfall des intraösophagealen $\mathrm{pH}$. In einer Untersuchung von 164 Patienten mit Refluxsymptomen ließ sich lediglich in $72 \%$ der Fälle eine pathologisch vermehrte Säure-Kontaktzeit im Ösophagus nachweisen [2]. In derselben Studie wiesen von 
Tab. 1 Extraösophageale Symptome und Erkrankungen, die refluxassoziiert sein können (adaptiert nach [17-19,62])

Heiserkeit im Rahmen einer posterioren Laryngitis

Globusgefühl

postnasal drip bei sekretorischer Pharyngitis bzw. chronischer Sinusitis behinderte Nasenatmung, purulentes Nasensekret im Rahmen einer chronischen Sinusitis

rezidivierende Otitis media

vocal cord dysfunction

trockener Reizhusten

Atemnot im Rahmen einer subglottischen Trachealstenose, einer

chronischen Bronchitis bzw. einer Lungenfibrose

asthmatische Exazerbation unklarer Ätiologie

35 untersuchten Asthmatikern, die keine Refluxsymptome angaben, $29 \%$ in der pH-Metrie einen signifikanten GÖR auf. Auch GÖR, der ohne typische Refluxsymptome einhergeht (engl. „silent reflux“) kann für diverse extraösophageale Beschwerden wie asthmatische Exazerbationen, Dysphonie, Retrosternalschmerz etc. verantwortlich sein [3].

Eine systematische Übersicht von 15 epidemiologischen Untersuchungen zur Häufigkeit des GÖR ergab eine Prävalenz von $10-20 \%$ in der westlichen Welt [4]. In Japan liegt die Prävalenz dagegen bei ca. 5\%. Für diesen Unterschied werden Ernährungsgewohnheiten und Übergewicht in den westlichen Industrienationen verantwortlich gemacht [5]. Eine eindeutige Geschlechtsspezifität des GÖR lässt sich nicht belegen.

\section{Diagnostik des GÖR}

Gelegentliches „Sodbrennen“, d.h. Sodbrennen, das nicht öfters als 1-2-mal pro Woche auftritt, erfordert keine weitergehende Diagnostik. Im Falle häufiger Beschwerden mit Beeinträchtigung der Lebensqualität, bei Therapieresistenz, d. h. Wiederauftreten von Refluxsymptomen nach Absetzen einer antisekretorischen Therapie, bei ösophagealen Komplikationen (Dysphagie etc.), als Screening im Hinblick auf ein mögliches Barrett-Syndrom und bei bestehenden Alarmsymptomen (Gewichtsverlust, Erbrechen, Anämie), ist eine Gastroskopie zum Ausschluss einer Stenose bzw. eines Malignoms indiziert [6]. Ein GÖR ohne makroskopische Zeichen einer Ösophagitis, die so genannte nicht erosive ösophageale Refluxkrankheit, lässt sich jedoch endoskopisch nicht objektivieren. Sie kann mittels einer 24-Stunden $\mathrm{pH}-$ Metrie, die allerdings invasiv ist und nicht überall zur Verfügung steht, nachgewiesen werden. Die Untersuchung ist dann indiziert, wenn nur eine dauerhafte medikamentöse Säurehemmung zur Beschwerdefreiheit führt und die Diagnose objektiviert werden soll, eine Fundoplicatio geplant ist, bzw. wenn extraösophageale Symptome beschrieben werden, die durch GÖR verursacht sein könnten [7] ( Tab. 1). Der prozentuale Anteil der Zeit mit einem $\mathrm{pH}<4$ ist das Maß mit der größten Sensitivität und Spezifität für die Diagnose einer Refluxkrankheit [8]. Die 95\%-Perzentile für die Dauer physiologischen Refluxes (pH $<4$ ) in der 24-Stunden-pH-Metrie beträgt 5,8\% [9]. Die 24-Stunden pH-Metrie gilt als der Goldstandard zum Nachweis des GÖR [10]. Bei der pH-Metrie wird der pH ca. $5 \mathrm{~cm}$ oberhalb des unteren Ösophagussphinkters gemessen. Manche pH-Metrie-Sonden sind mit einer zweiten pH-Elektrode im proximalen Ösophagus ausgestattet, um GÖR, der bis in den Pharynxbereich gelangt, registrieren zu können. Die meisten pH-Metrie-Systeme verfügen über eine Software, die es erlaubt, das Auftreten klinischer Symptome zu markieren. Treten spezifische Symptome in Assoziation (nach einer Latenz von 0-5 Minuten) mit Refluxepisoden auf, wird ein kausaler Zusammenhang angenommen. Wie eine Untersuchung von Irwin u. Mitarb. [11] ergab, muss von einem refluxinduzierten chronischen Husten auch dann ausgegangen werden, wenn in weniger als 5,8\% der Messperiode GÖR nachgewiesen wird, nachgewiesene Refluxepisoden aber überwiegend mit Husten assoziiert sind.

Die Messung des pH-Wertes im Kondensat der Ausatemluft, eine neue, nicht invasive Methode GÖR nachzuweisen, befindet sich in klinischer Erprobung [12].

\section{Ösophageale und extra-ösophageale Komplikationen des GÖR}

GÖR kann zu einer Refluxösophagitis mit der Gefahr einer Stenosierung, Blutung etc. führen. Bei $5 \%$ der Refluxkranken entwickelt sich eine Epithelmetaplasie (Barrett-Metaplasie), die mit dem Risiko einer malignen Entartung (Adenokarzinom des Ösophagus) behaftet ist.

Sowohl subjektiv wahrgenommener GÖR, als auch GÖR ohne typische Refluxsymptome, vermag atypische Symptome und extraösophageale Erkrankungen auszulösen bzw. solche zu komplizieren. GÖR gilt beispielsweise als wichtige Differenzialdiagnose des Angina pectoris-Schmerzes.

Chronischer Husten stellt eine typische extraösophageale Komplikation von GÖR dar [13,14]. Eine medikamentöse Säurehemmung bei chronischem Husten mit nachgewiesenem GÖR führt oft zu einer Besserung. Der Erfolg liegt aber deutlich unter $100 \%$, da extraösophageale Refluxsymptome häufig einer intensiveren und längeren Therapie als alleiniges Sodbrennen bedürfen und chronischer Husten in vielen Fällen durch mehr als einen kausalen Faktor bedingt wird. Irwin u. Mitarb. [15] konnten zeigen, dass chronischer Reizhusten in den allermeisten Fällen durch GÖR, postnasal drip im Rahmen einer chronischen Sinusitis und/ oder eine bronchiale Hyperreaktivität ausgelöst wird, sofern ein aktuelles Thorax-Röntgenbild unauffällig ist, kein Nikotinabusus betrieben wurde bzw. in den letzten sechs vorangehenden Wochen kein ACE-Hemmer eingenommen worden war.

Ing u. Mitarb. [16] wiesen durch Instillation von Säure (Bernstein-Test) versus isotonischer Kochsalzlösung in den Ösophagus nach, dass die Präsenz von Säure im Ösophagus bei Patienten mit chronischem Reizhusten sofortige Hustenattacken auslöst. Die lokale Gabe von Lidocain vor der Säureapplikation inhibierte den Husten. Mit der ösophagealen Applikation von Ipratropiumbromid gelang dies nicht. Wurde Ipratropiumbromid dagegen inhaliert, konnten die säureassoziierten Hustenattacken unterdrückt werden. Diese Daten stützen die These, dass GÖR Husten indirekt durch einen vagalen, ösophageal-tracheobronchialen Reflex vermittelt („Reflexhypothese“).

GÖR kann jedoch auch direkt extraösophageale Organe schädigen, da im Rahmen einer Refluxkrankheit Magensekret teilweise bis in die Atemwege gelangt („Aspirationshypothese“). GÖR wird deshalb im HNO-Bereich für multiple Probleme verantwortlich gemacht [17]. Im Wesentlichen handelt es sich dabei um die posteriore Laryngitis mit Heiserkeit, Stimmbandgranulome und Globusgefühl. Es wird angenommen, dass GÖR auch an der Entstehung der sekretorischen Pharyngitis (DD postnasal drip!), der chronischen Sinusitis und vor allem bei Kindern an rezidivierenden Otitiden beteiligt sein kann (Übersicht in [18]). Auch bei der 
vocal cord dysfunction wird eine kausale Bedeutung diskutiert [19]. Der pharyngeale Unterdruck während Apnoephasen bei der obstruktiven Schlafapnoe kann zur Aspiration von Säure aus dem Magen führen. Eine CPAP-Therapie erweist sich dann als therapeutisch wirksam [20].

Ein massiver GÖR, der noch proximal im Ösophagus und auch in aufrechter Körperhaltung nachweisbar ist [21], dringt unter Umständen bis in die unteren Atemwege vor und führt dort zu Schleimhautreaktionen bzw. -schädigungen [22]. Eine ursächliche Beteiligung von GÖR bei manchen Formen der subglottischen Trachealstenose, der chronischen obstruktiven Bronchitis [23] und der idiopathischen Lungenfibrose [24] wird vermutet. Selbst im Falle einer adäquaten Säurehemmung ist es denkbar, dass gallensäurehaltiges Sekret aus Magen und Dünndarm in Form von Mikroaspirationen die Atemwege erreicht. Derartiger „nicht azider“ GÖR dürfte ebenfalls in der Lage sein, die Atemwege zu reizen oder zu schädigen.

Eine aktuelle, große Kohortenstudie aus Norwegen, die über 65000 Personen einschloss, ergab eine ausgeprägte Assoziation zwischen starker Atemnot (unabhängig von deren Ätiologie) und intensiven, wiederkehrenden Refluxsymptomen. Die odds ratio betrug 12 (95\% CI 9.5-15.2) [25].

\section{GöR und Asthma bronchiale}

Schon William Osler [26] wies 1892 darauf hin, dass zwischen GÖR und Asthma kausale Zusammenhänge bestehen. Diese Kausalitätsvermutung wurde in einem Positionspapier der American Gastroenterological Association als nicht evidenzbasiert infrage gestellt [27].

Durch multiple Studien ist belegt, dass GÖR bei Asthmatikern viel häufiger als bei Gesunden auftritt. Messungen mittels 24-Stunden-pH-Metrie wiesen bei 36\% [1] bis 80\% [28] der untersuchten Asthmatiker GÖR nach. Verschiedene Gründe könnten diese Häufung erklären:

Theophyllin und oral applizierte Betastimulatoren üben eine relaxierende Wirkung auf den unteren Ösophagussphinkter aus [29,30], bei inhalativen Betastimulatoren ist die Wirkung dosisabhängig [31,32]. Es existiert allerdings keine Studie, die belegt, dass die vermehrte Sphinkterrelaxation auch zu einer Zunahme von GÖR führt. So fand sich in einer plazebokontrollierten Untersuchung, bei der unter Depot-Theophyllin $\mathrm{pH}-$ Metrien durchgeführt worden waren, kein vermehrter GÖR [33].

In hohen Dosen verstärken systemische Kortikosteroide GÖR bei Asthmatikern [34].

Die Überblähung der Lunge im Rahmen eines Volumen pulmonum auctum bedingt eine Erhöhung des intraabdominellen Drucks, was die Entstehung von GÖR fördern kann. Die Relevanz eines tief stehenden Zwerchfells im Hinblick auf die Manifestation des GÖR bleibt aber unklar, da auch bei Patienten mit Lungenfibrose, die meist einen Zwerchfellhochstand aufweisen, ein GÖR gehäuft nachweisbar ist.

Im Rahmen von Methacholin-Tests wurde beobachtet, dass mit zunehmender bronchialer Obstruktion vermehrt TLESR mit konsekutivem GÖR auftreten [35].

Im Hinblick auf die Wechselwirkung von GÖR und Asthma sind folgende Fragen von klinischer Bedeutung:

1. Können auch Asthmatiker im Falle von GÖR einen Reizhusten entwickeln, ähnlich wie dies bei Nichtasthmatikern vorkommt, ohne dass zwangsläufig eine vermehrte asthmati- sche Aktivität vorliegt, die einer intensiveren antiasthmatischen Therapie bedarf?

2. Stellt GÖR einen Co-Faktor dar, der zur Manifestation eines Asthmas beiträgt?

3. Vermag ein massiver GÖR ein Asthma derart zu komplizieren, dass es medikamentös schlechter kontrollierbar wird, bzw.

dass durch GÖR vermehrt asthmatische Exazerbationen auftreten?

ad 1) Es liegt nahe, dass GÖR bei Patienten mit Asthma, noch mehr als bei Menschen ohne bronchiale Hyperreagibilität, Husten auslösen kann. 24-Stunden pH-Metrie-Protokolle ergaben, dass bei Asthmatikern über 50\% aller Hustenepisoden mit GÖR korrelierten $[2,36]$. Es gibt aber keinen Hinweis dafür, dass refluxassoziierter Husten bei einem Asthmatiker zwangsläufig als Asthmasymptom gewertet werden müsste, besonders dann, wenn sonst klinisch keine Hinweise für eine unzureichende Asthmakontrolle vorliegen. In dieser Hinsicht ist es wichtig zu wissen, dass sich Husten, der durch GÖR ausgelöst wird, nicht durch ein spezifisches zircadianes Muster auszeichnet [37], sondern auch tagsüber auftritt. Hierzu passt, dass GÖR mit extraösophagealen Symptomen entgegen der üblichen Meinung vor allem in aufrechter Position und im Wachzustand nachweisbar ist [38].

ad 2) GÖR vermag über vagale Reflexe Husten und vermutlich über den gleichen Reflexbogen auch Bronchospasmen [39] auszulösen. In älteren Studien war bei Patienten mit nicht-allergischem Asthma in bis zu 90\% der Fälle GÖR beschrieben worden [40]. Diese Befunde legten nahe, dass pathologischer GÖR im Falle einer genetisch determinierten bronchialen Hyperreagibilität zur Manifestation eines Asthmas beitragen könnte. Eine aktuelle britische Kohortenstudie, die über 5000 Patienten mit primärer Diagnose GÖR und knapp 10000 Patienten mit primärer Diagnose Asthma bronchiale einschloss, ergab jedoch, dass für Menschen mit GÖR das Risiko an Asthma zu erkranken, nicht signifikant zunimmt (relatives Risiko 1.2, 95\% CI 0.9-1.6), für Patienten mit Asthma dagegen das Risiko GÖR zu entwickeln, gering erhöht ist (relatives Risiko 1.5, 95\% CI 1.2 - 1.8) [41].

ad 3) Experimentell wurde gezeigt, dass bei Asthma durch Instillation von Säure in den Ösophagus die bronchiale Hyperreagibilität zunimmt [42] und dass spontaner proximaler GÖR bei Asthmatikern via Mikroaspirationen teilweise zu einem Abfall des intratrachealen $\mathrm{pH}$ führt mit simultaner Zunahme der bronchialen Obstruktion [43]. Diese Befunde lassen darauf schließen, dass GÖR ein vorbestehendes Asthma komplizieren kann. Klinische Studien zu dieser Fragestellung ergeben jedoch kontroverse Ergebnisse:

In einer Arbeit von Irwin u. Mitarb. [3] aus dem Jahr 1993, als in den USA hoch dosierte topische Steroide noch nicht einen Grundpfeiler der Therapie des schweren Asthmas darstellten, wurde GÖR als der wichtigste kausale Faktor im Falle einer Therapieresistenz bei Asthma identifiziert. In einer aktuellen Studie, die 136 Patienten mit schwer kontrollierbarem Asthma einschloss, korrelierte GÖR - gemeinsam mit fünf anderen Einzelfaktoren - gehäuft mit rezidivierenden asthmatischen Exazerbationen [44]. Eine Metaanalyse zur Refluxtherapie bei Asthma, die überwiegend noch Studien mit H2-Blockern enthielt, ergab eine Besserung von Asthmasymptomen und eine Reduktion des Bedarfs an Antiasthmatika, jedoch keine Verbesserung der Lungenfunktion [45].

Heaney u. Mitarb. [46] und Leggett u. Mitarb. [47] stellten diese Ergebnisse in eigenen Untersuchungen von 73 bzw. 52 Patienten mit schwer kontrollierbarem Asthma infrage. In beiden unter- 
suchten Kollektiven aus Belfast, die möglicherweise z. T. die gleichen Patienten enthielten, suchte man nach Exazerbationsfaktoren, die dann spezifisch behandelt wurden. Im Falle des Nachweises von GÖR erfolgte die Applikation von ProtonenpumpenInhibitoren (PPI). Bei den Patienten, die sich auf eine therapeutische Intervention verbesserten, bzw. bei denen, die kein Ansprechen zeigten, war die Häufigkeit von GÖR mit gut $50 \%$ identisch. Auch bei Patienten mit weniger schwerem Asthma lässt die Refluxtherapie mit PPI statistisch keine relevante Verbesserung der Asthmasymptome bzw. keine Reduktion des Bedarfs an Antiasthmatika erkennen $[48,49]$. Eine Cochrane-Metaanalyse, die 2003 publiziert worden ist, ergab ebenfalls keine generelle Verbesserung des Asthmas unter säurehemmender Therapie [50]. Die aufgeführten Untersuchungen, die keinen positiven Effekt einer Säurehemmung auf das Asthma ergaben, müssen jedoch mit Vorbehalt beurteilt werden. Milkes u. Mitarb. [51] zeigten mithilfe von pH-Metrien, dass über 50\% der Patienten mit GÖR auch unter einer Standardtherapie mit PPI noch aziden GÖR aufwiesen. Der Einsatz der intraluminalen Mehrkanal-ImpedanzMessung bei simultaner pH-Metrie ergab, dass trotz medikamentöser Säurehemmung noch GÖR auftritt [52], teilweise jedoch mit normalem pH-Wert. Da anzunehmen ist, dass nicht nur die Säure via vagaler Reflexe, sondern auch nicht-azider GÖR via Mikroaspirationen die unteren Atemwege reizen können, stellt die alleinige Säurehemmung mit PPI vermutlich nicht immer eine ausreichende Refluxtherapie dar. Entsprechend ließen operative Therapieverfahren zur Therapie eines pathologischen GÖR teilweise bessere Resultate als eine alleinige medikamentöse Behandlung mit Säureblockern erkennen [53]. Das ist verständlich, da nur eine erfolgreiche Fundoplicatio, nicht aber eine medikamentöse Säurehemmung die physiologische Antirefluxbarriere wieder herstellt. Entsprechend ließ sich in einer Studie bei Kindern mit Asthma und GÖR, bei der ergänzend zur PPI-Therapie großzügig die Indikation zur Medikation mit Prokinetika bzw. zur Fundoplicatio gestellt worden war, eine Reduktion der Medikation mit Antiasthmatika erzielen [54]. In kontrollierten Studien, die eine Kombination von Prokinetika und PPI gegen PPI alleine verglichen, ergab sich für die Kombination allerdings kein Vorteil [55,56].

Aus den widersprüchlichen Ergebnisse der verschiedenen Untersuchungen kann als minimale Quintessenz gefolgert werden, dass GÖR nicht generell ein vorhandenes Asthma verschlechtert. Es muss aber davon ausgegangen werden, dass GÖR bei einzelnen Patienten asthmatische Exazerbationen bzw. Therapieresistenz auszulösen vermag. Auch die erwähnte Cochrane-Metaanalyse ergab, dass Subgruppen von Patienten von einer PPI-Therapie profitieren [50]. Es gelang in der Metaanalyse aber nicht, anhand potenzieller Refluxsymptome, diese Patienten genauer zu charakterisieren. Eine Schwäche dieser Metaanalyse war, dass GÖR nicht objektiv, sondern nur anhand von Symptomen mit der bekannten mäßigen Sensitivität und Spezifität nachgewiesen worden war. Harding u. Mitarb. [57] konnten jedoch nachweisen, dass sich asthmatische Symptome dann unter einer Therapie mit Omeprazol verbessern, wenn anamnestisch Regurgitationen und/oder der Nachweis von proximalem GÖR in der pHMetrie vorlagen. Eine ganz aktuelle Untersuchung von Kiljander u. Mitarb. [58], in der randomisiert und plazebokontrolliert über 16 Wochen täglich $80 \mathrm{mg}$ Esomeprazol eingesetzt wurde, zeigte nur für die Gruppe der Asthmatiker mit GÖR, die trotz antiasthmatischer Therapie noch unter nächtlichen Asthmasymptomen litten, eine klare Verbesserung des morgendlichen Peak flows.

\section{Diagnostik und Therapie des GÖR beim Asthma}

Das Auftreten von GÖR bei Asthmatikern muss differenziert betrachtet werden. Klinisch lassen sich drei Szenarien unterscheiden:

1.) Refluxsymptome, die bei einem Asthmatiker auftreten, dessen Asthma medikamentös gut eingestellt ist, erfordern dieselben therapeutischen Interventionen wie bei einem Nicht-Asthmatiker, d.h. das Asthma bleibt bei der Refluxtherapie unberücksichtigt.

2.) Das klinische Bild ist anders zu beurteilen, wenn ein Asthmatiker einen trockenen Reizhusten entwickelt, sonst aber keine klinischen Symptome einer vermehrten asthmatischen Aktivität vorliegen. Als Hauptsymptom einer erhöhten asthmatischen Aktivität muss vor allem vermehrte Atemnot tagsüber, besonders bei körperlicher Aktivität, angesehen werden [59]. Durch den Einsatz lang wirkender Betastimulatoren wird nächtliches Erwachen mit Husten und Atemnot, was allgemein als frühes Zeichen einer beginnenden Exazerbation galt, lange Zeit unterdrückt [60].

Wenn eine Therapieintensivierung des Asthmas im Falle vermehrten Hustens keine Besserung der Symptome ergibt, keine floride Sinusitis nachweisbar ist, kein ACE-Hemmer eingenommen wird und das Röntgenbild des Thorax unauffällig ist, wird eine probatorische Therapie mit einem PPI in Standarddosis empfohlen. Spricht der Husten nach vier- bis achtwöchiger Gabe [61] auf die Säurehemmung an, kann analog zur Situation bei Nichtasthmatikern von einem refluxassoziierten Husten ausgegangen werden. Kommt es unter dieser Therapie zu keiner Besserung, muss der Husten jedoch primär als Asthma-Äquivalent gewertet werden. Da sich das Symptom auf die initiale Intensivierung der Asthmatherapie nicht gebessert hat, wird das Asthma als „schlecht kontrollierbar“ eingestuft und entsprechend verfahren (vgl. Punkt 3). Die genannte Empfehlung gilt sowohl für Asthmatiker mit Reizhusten, bei denen typische Refluxsymptome vorliegen, als auch für solche, bei denen diese Symptome fehlen.

3.) Im Falle eines trotz stadiengerechter Therapie symptomatischen, d.h. therapeutisch schlecht kontrollierten Asthmas, bei dem anamnestisch Hinweise für GÖR [62] (s. auch ○ Tab. 1) zu eruieren sind, schlagen wir den folgenden diagnostischen und therapeutischen Algorithmus ( Abb. 1) vor, mit dessen Hilfe ein refluxassoziiertes Asthma ausgeschlossen bzw. bewiesen werden kann. Der Algorithmus basiert darauf, dass Patienten mit therapierefraktärem Asthma zuallererst hinsichtlich Therapietreue (Compliance) und korrekter Inhalationstechnik evaluiert und geschult werden müssen. Können diese beiden Faktoren als Ursache der Therapieresistenz ausgeschlossen werden, sollte bei Patienten mit anamnestischen Hinweisen für GÖR ein Therapieversuch mit PPI (40-80 mg) unternommen werden, dessen Dauer sich auf mindestens 8 Wochen beläuft $[27,58]$.

Fehlen offensichtliche Hinweise auf GÖR, ist eine Abklärung der Therapieresistenz entsprechend den Empfehlungen von Menz u. Mitarb. [63] indiziert. Für den Fall, dass diese Abklärungen keinen anderen plausiblen Grund für die Therapieresistenz ergeben, ist wegen der Möglichkeit eines GÖR ohne typische Refluxsymptome auch dann eine PPI-Therapie über mindestens 8 Wochen unerlässlich.

Selbst im Falle der kompletten Elimination von Refluxsymptomen durch PPI werden in bis zu 50\% der Fälle noch pathologische intraösophageale pH-Werte gemessen [51]. Dies impliziert, dass bei Verdacht auf refluxassoziiertes Asthma, auch im Falle 


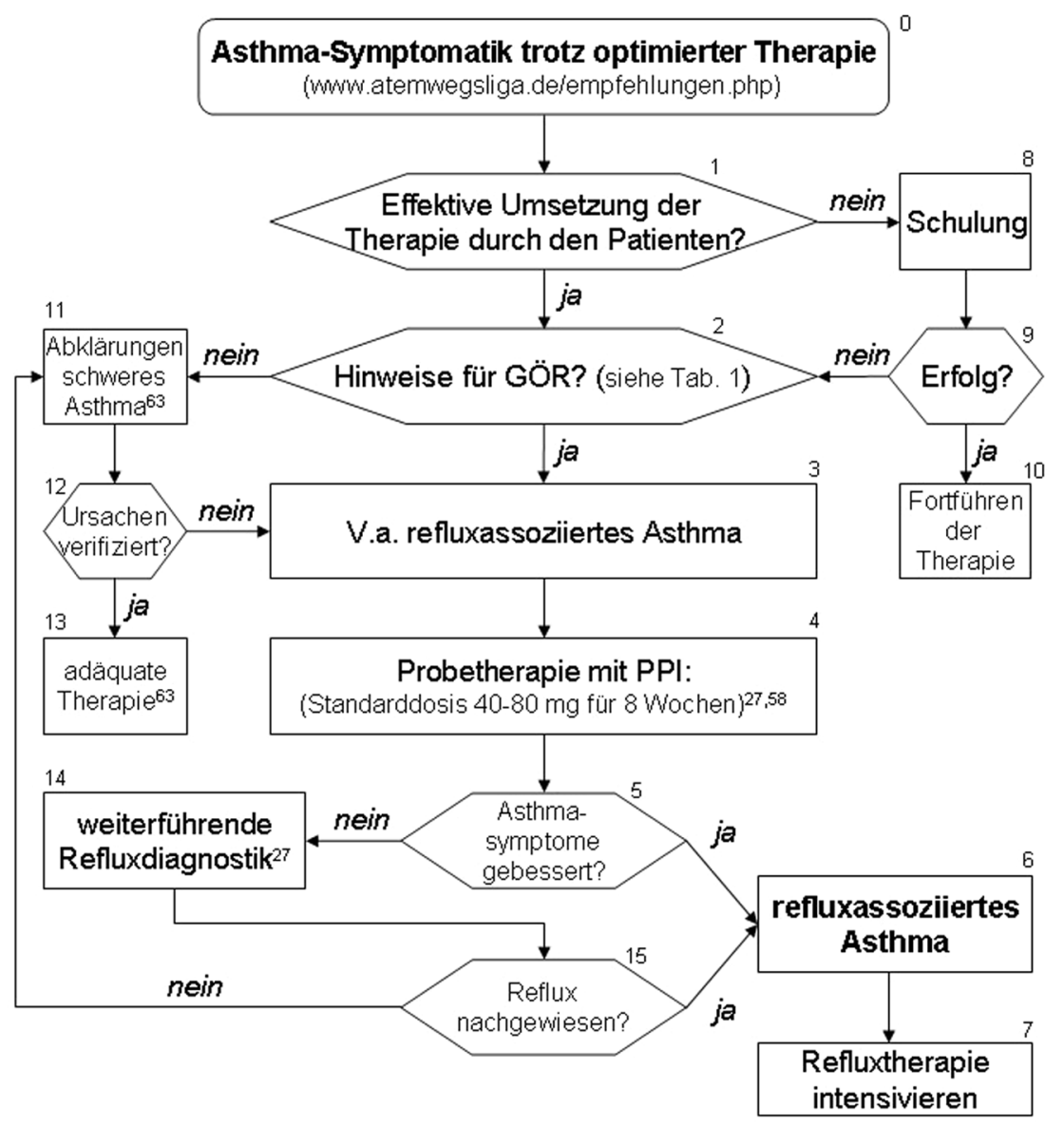

Abb. 1 Algorithmus: Diagnostik bei Asthma und GÖR. der Persistenz von Asthmasymptomen unter PPI-Therapie, der Algorithmus weitere Abklärungen hinsichtlich aziden bzw. nicht-aziden GÖR (24-Stunden-pH-Metrie, intraluminale Mehrkanal-Impedanz-Messung...) vorsehen muss ( Abb. 1).

Lässt sich ein refluxassoziiertes Asthma nachweisen, das trotz adäquater antiasthmatischer Therapie und Gabe von PPI in Standarddosierung symptomatisch bleibt, sollte die weitere Abklärung (z.B. mittels 24-Stunden-pH-Metrie) bzw. mögliche Intensivierung der Refluxtherapie (höhere PPI-Dosen) in Absprache mit einem Gastroenterologen erfolgen.

\section{Nicht-medikamento̊se Therapie des GöR}

Die Pharmakotherapie des GÖR wird durch nicht-medikamentöse Maßnahmen ergänzt. Obgleich der Wert der im Folgenden aufgelisteten Empfehlungen nicht durch entsprechende Studien belegt ist, wird generell Adipösen eine Gewichtsreduktion empfohlen. Veränderungen des Lebensstils sind häufig notwendig, u. a. Erlernen von Techniken zur Stressbewältigung sowie Nikotin- und Alkoholkarenz. Bei den Hauptmahlzeiten sollte der Magen nicht überfüllt werden. Auf Mahlzeiten, die später als zwei Stunden vor der Bettruhe erfolgen, ist zu verzichten. Verschiedene Autoren empfehlen, dass nachts das Kopfteil des Bettes höher gestellt werden soll. Dafür liegt aber keine klare Evidenz vor $[64,65]$, vor allem weil die Motilität des Ösophagus ein aktiver Prozess ist, d.h. der Einfluss der Schwerkraft vermutlich gering sein dürfte. Dennoch „schwören“ viele Patienten auf diese Maßnahme. Nahrungsmittel (Pfefferminz, Koffein und stark zuckerbzw. fetthaltige Speisen), die den unteren Ösophagussphinkter relaxieren lassen, sind ebenfalls zu meiden. Um Patienten Alternativen zu ihren bisherigen Essgewohnheiten aufzuzeigen, ist eine Ernährungsberatung, die Instruktionen hinsichtlich langsamen Essens und guten Kauens beinhaltet, sinnvoll. Obwohl die wissenschaftliche Evidenz dafür fehlt, wird bei Patienten mit refluxassoziiertem Asthma versucht werden, auf orale Betastimulatoren und Theophyllinpräparate zu verzichten.

\section{Institutsangaben}

1 Luzerner Höhenklinik Montana, CH

Pneumologie, Johannes Gutenberg-Universität Mainz

Pneumologie, Robert-Koch-Klinik Leipzig

${ }^{4}$ Pneumologie/Allergologie-Zentrum Krankenhaus Maingau, Frankfurt/Main

Dept. Pulmology, University Leiden, NL

Pneumologie-Praxis Ulm

Pneumologie, Philipps-Universität Marburg

Medizinische Klinik Fürth

${ }^{9}$ Gastroenterologie, Philipps-Universität Marburg

${ }^{10}$ Hochgebirgsklinik Davos, Schweiz

\section{Literatur}

1 Kiljander TO, Laitinen JO. The prevalence of gastroesophageal reflux disease in adult asthmatics. Chest 2004; 126: 1490-1494

2 Harding SM, Guzzo MR, Richter JE. 24 hour esophageal pH testing in asthmatics. Respiratory symptom correlation with esophageal acid events. Chest 1999; 115: 654

3 Irwin RS, Curley FJ, French CL. Difficult to control asthma: Contributing factors and outcome of a systematic management protocol. Chest 1993; 103: 1662

4 Dent J, El-Serag HB, Wallander MA et al. Epidemiology of gastrooesophageal reflux disease: a systematic review. Gut 2005; 3: 543

5 Fass R, Quan SF, O'Connor GT et al. Predictors of heartburn during sleep in a large prospective cohort study. Chest 2005; 127: 1658-1666 
6 Koop H, Schepp W, Müller-Lissner S et al. Gastroösophageale Refluxkrankheit - Ergebnisse einer evidenzbasierten Konsensuskonferenz der Deutschen Gesellschaft für Verdauungs- und Stoffwechselkrankheiten. Z Gastroenterol 2005; 43: 163 - 194 (www.dgvs.de)

7 Empfehlungen der Deutschen Gesellschaft für Neurogastroenterologie und Motilität. 24-Stunden-Ösophagus-pH-Metrie. www.neurogastro.de/empfehlungen

8 Schindlbeck NE, Ippisch H, Klauser AG et al. Which pH threshold is best in esophageal pH monitoring. Am J Gastroenterol 1991; 86: 1138 1141

9 Jamieson JR, Stein HJ, DeMeester TR et al. Ambulatory 24-h esophageal pH monitoring: normal values, optimal threshold, specificity, sensitivity, and reproducibility. Am J Gastroenterol 1992; 87: 1102 - 1111

10 Kahrilas PJ, Quigley EM. Clinical esophageal pH recording: a technical review for practice guideline development. Gatroenterology 1996; 110: 1982 - 1996

11 Irwin RS, French CL, Curley FJ. Chronic cough due to gastroesophageal reflux: clinical, diagnostic, and pathogenetic aspects. Chest 1993; 104: $1511-1511$

12 Hunt J, Yu Y, Burns J et al. Identification of acid reflux cough using serial assays of exhaled breath condensate $\mathrm{pH}$. Cough 2006; $2: 3$

13 Kardos P, Cegla U, Gillissen A et al. Leitlinie der Deutschen Gesellschaft für Pneumologie zur Diagnostik und Therapie von Patienten mit akutem und chronischem Husten. Pneumologie 2004; 58: 570-602

14 Chang AB, Lasserson TJ, Kiljander TO et al. Systematic review and metaanalysis of randomised controlled trials of gastro-oesophageal reflux interventions for chronic cough associated with gastro-oesophageal reflux. BMJ 2006; 332: 11 - 14

15 Irwin RS, Curley FJ, French CL. Chronic cough: The sprectrum and frequency of causes, key components of the diagnostic evaluation, and outcome of specific therapy. Am Rev Respir Dis 1990; 141: 640-647

16 Ing AJ, Ngu MC, Breslin AB et al. Pathogenesis of chronic cough associated with gastroesophageal reflux. Am J Respir Crit Care Med 1994; 149: 160 - 167

17 Wong RK, Hanson DG, Waring PJ et al. ENT manifestations of gastroesophageal reflux. Am J Gastroenterology 2000; 95 (Suppl. 8): S15S22

18 Poelmans J, TackJ. Extraesophageal complications of gastroesophageal reflux. Gut 2005; 54: $1492-1499$

19 Powell DM, Karanfilov BI, Beechler KB et al. Paradoxical vocal cord dysfunction in juveniles. Arch Otolaryngol Head Neck Surg 2000; 126 : $29-34$

20 Kerr P, Shoenut JP, Millar T et al. Nasal CPAP reduces gastroesophageal reflux in obstructive sleep apnea syndrome. Chest 1992; 101: 15391544

21 Harding SM, Guzzo MR, Richter JE. The prevalence of gastroesophageal reflux in asthma patients without reflux symptoms. Am Rev Respir Crit Care Med 2000; 162: 34-39

22 Ruth M, Carlsson SM, Haile JM et al. Scintigraphic detection of gastroesophageal aspiration in patients with respiratory disorders. Clin Physiol 1993; 13: 19-33

23 Mohklesi B, Morris AL, Huang CF et al. Increased prevalence of gastroesophageal reflux in patients with COPD. Chest 2001; 119: 10431048

24 Tobin RW, Pope CE, Pellegrini CA et al. Increased prevalence of gastroesophageal reflux in patients with idiopathic pulmonary fibrosis. Am Rev Respir Crit Care Med 1998; 158: 1804-1808

25 Nordenstedt $H$, Nilson $M$, Johansson $S$ et al. The relation between gastroesophageal reflux and respiratory symptoms in a populationbased study. Chest 2006; 129: 1051 - 1056

26 Osler WB. Bronchial asthma. In: The principles and practice of medicine. New York: NY. Appleton, 1892: 497-501

27 Peterson WL. American gastroenterological association consensus development panel: improving the management of GERD (http://www.gastro.org/user-assets/documents/GERDmonograph.pdf)

28 Sontag SJ, O'Connell S, Khandelwal T et al. Most asthmatic patients have gastroesophageal with or without bronchodilator therapy. Gastroenterology 1990; 99: 613-620

29 Stein MR, Towner TG, Weber RW et al. The effect of theophylline on the lower esophageal sphincter pressure. Ann Allergy 1980; 45: 238 - 241

30 DiMarino AJ, Cohen S. Effect of an oral beta-2 adrenergic agonist on lower esophageal sphincter pressure in normal and in patients with achalasia. Dig Dis Sci 1982; 27: 1063 - 1066
31 Crowell MD, Zayat EN, Lacy BE et al. The effects of an inhaled beta2-adrenergic agonist on lower esophageal function. Chest 2001; 120: $1184-1189$

32 Michoud MC, Leduc T, Proulx F et al. Effect of salbutamol on gastroesophageal reflux in healthy volunteers and patients with asthma. J Allergy Clin Immunol 1991; 87: 762 - 767

33 Huber D, Gaudric M, Guerre J et al. Effect of theophylline on gastroesophageal reflux in patients with asthma. J Allergy Clin Immunol 1988; 81: $1168-1174$

34 Lazenby JP, Guzzo MR, Harding SM et al. Oral corticosteroids increase esopagheal contact times in patients with asthma. Chest 2002; 121 : $625-634$

35 Zerbib F, Guisset O, Lamouliatte $\mathrm{H}$ et al. Effects of bronchial obstruction on lower esophageal sphincter motility and gastroesophageal reflux in patients with asthma. Am J Respir Crit Care Med 2002; 166: 1206 1211

36 Avidan B, Sonnenburg A, Schnell TG et al. Temporal associations between coughing or wheezing and cid reflux in asthmatics. Gut 2001; 49: $767-772$

37 Mello CJ, Irwin RS, Curley FJ. Predictive values of the character, timing, and complications of chronic cough in diagnosing its cause. Arch Intern Med 1996; 156: 997-1003

38 Irwin RS, Zawacki JK, Curlwy FJ et al. Chronic cough as the sole presenting manifestation of gastroesophageal reflux. Am Rev Respir Dis 1989; 140: $1294-1300$

39 Spaulding Jr HS, Mansfield LE, Stein MR et al. Further investigation of the association between gastroesophageal reflux and bronchoconstriction. J Allergy Clin Immunol 1982; 69: 516-521

40 Larrain A, Carrasco E, Galleguillos $F$ et al. Medical and surgical treatment of nonallergic asthma associated with gastroesophageal relux. Chest 1991; 99: 1330-1335

41 Ruigomez A, Rodriguez LA, Wallander M-A et al. Gastroesophageal reflux disease and asthma: A longitudinal study in UK general practice. Chest 2005; 128: 85-93

42 Herve $P$, Denjeen A, Jian $R$ et al. Intraesophageal perfusion of acid increases the bronchomotor response to methacholine and to isocapnic hyperventilation in asthmatic subjects. Am Rev Respir Dis 1986; 134: 986-989

43 Jack CI, Calverley PM, Donelly RJ et al. Simultaneous tracheal and esophageal $\mathrm{pH}$ measurements in asthmatic patients with gastroesophageal reflux. Thorax 1995; 50: $201-204$

44 ten Brinke A, Sterk PJ, Masclee AA et al. Risk factors of frequent exacerbations in difficult-to-treat-asthma. Eur Respir J 2005; 26: 812 - 818

45 Field SK, Sutherland LR. Does medical antireflux therapy improve asthma in asthmatics with gastroesophageal reflux? A critical review of the literature. Chest 1998; 114: 274-283

46 Heaney LG, Conway E, Kelly C et al. Predictors of therapy resistant asthma: outcome of a systematic evaluation protocol. Thorax 2003; 58: $561-566$

47 Leggett JJ, Johnston BT, Mills M et al. Prevalence of gastroesophageal reflux in difficult asthma. Relationship to asthma outcome. Chest 2005; 127: $1227-1231$

48 Littner MR, Leung FW, Ballard ED et al. Effects of 24 weeks of lansoprazole therapy on asthma symptoms, exazerbations, quality of lief, and pulmonary function in adult asthmatic patients with acid reflux symptoms. Chest 2005; 128: $1128-1135$

49 Størdal K, Johannesdottir GB, Bentsen BS et al. Acid suppression does not change respiratory symptoms in children with asthma and gastroesophageal reflux disease. Arch Dis Child 2005; 90: 956-960

50 Gibson PG, Henry HL, Coughlan JL. Gastro-oesophageal reflux treatment for asthma in adults and children. Syst Rev 2003; (2): CDOOI1496

51 Milkes D, Gerson LB, Triadafilopoulos G. Complete elimination of reflux symptoms does not guarantee normalization of intraesophageal and intragastric $\mathrm{pH}$ in patients with gastroesophageal reflux disease. Am J Gastroenterology 2004; 99: 991 - 996

52 Vela MF, Camacho-Lobato L, Srinivasan $R$ et al. Simultaneous intraesophageal impedance and $\mathrm{pH}$ measurement ofacid and nonacid gastroesophageal reflux: effect of omeprazole. Gastroenterology 2001; 120: $1599-1606$

53 Sontag SJ, O'Connell S, Khandelwal S et al. Asthmatics with gastroesophageal reflux: long term results of a randomized trial of medical and surgical antireflux therapies. Am J Gastrenterology 2003; 98: $987-$ 999 
54 Khoshoo V, Le T, Haydel M. Role of gastroesophageal reflux in older children with persistent asthma. Chest 2003; 123: 1008-1013

55 Rensburg CJ van, Bardhan KD. No clinical benefit of adding cisapride to pantoprapzole for treatment of gastro-oesophageal reflux disease. Eur J Gastroenterol Hepatol 2001; 13: 909-914

56 Smythe A, Bird NC, Troy GP et al. Does the addition of a prokinetic to proton pump inhibitor therapy help reduce duodenogastro-oesophageal reflux in patients with Barrett's oesophagus? Eur J Gastroenterol Hepatol 2003; 15: 305-312

57 Harding SM, Richter JE, Guzzo MR. Asthma and gastroesophageal reflux: Acid suppressive therapy improves asthma outcome. Am J Med 1996; 100: $395-405$

58 Kiljander TO, Harding SM, Field SK et al. Effects of Esomeprazole $40 \mathrm{mg}$ twice daily on asthma. A randomized Plazebo-controlled trial. Am J Respir Crit Care Med 2006; 173: 1091 - 1097

59 Dennis SM, Altmann DR, Lee TH et al. Increase in daytime symptoms is a sensitive and specific criterion for predicting corticosteroid-treated exacerbations in a clinical asthma trial. Clin Exp Allergy 2005; 35: $308-312$

60 Mclvor RA, Pizzichini E, Turner MO et al. Potential masking effect of salmeterol on airway inflammation in asthma. Am J Respir Crit Care Med 1998; 158: $924-930$

61 Poe RH, Kallay MC. Chronic cough and gastroesophageal reflux disease. Experience with specific therapy for diagnosis and treatment. Chest 2003; 123: 679-684

62 Fox M, Forgacs I. Gastro-oesophageal reflux disease. BMJ 2006; 332 $88-93$

63 Menz G, Buhl R, Gillissen A et al. Schwieriges Asthma: Klinische Phänotypen und Prinzipien der Therapie. Pneumologie 2002; 56: 132 - 137

64 Tobin JM, McCloud P, Cameron DJ. Posture and gastro-oesophageal reflux: a case for left lateral positioning. Arch Dis Child 1997; 76: $254-$ 258

65 Johnson LF, DeMeesters TR. Evaluation of elevation of the head of the bed, bethanechol, and antacid form tablets on gastroesophageal reflux. Dig Dis Sci 1981; 26: 673-680 